Louisiana State University

LSU Digital Commons

Faculty Publications

Department of Physics \& Astronomy

$12-1-2005$

\title{
MEGA: A Medium-Energy Gamma-ray Astronomy mission concept
}

P. F. Bloser

University of New Hampshire Durham

J. M. Ryan

University of New Hampshire Durham

M. L. McConnell

University of New Hampshire Durham

J. R. Macri

University of New Hampshire Durham

U. Bravar

University of New Hampshire Durham

See next page for additional authors

Follow this and additional works at: https://digitalcommons.Isu.edu/physics_astronomy_pubs

\section{Recommended Citation}

Bloser, P., Ryan, J., McConnell, M., Macri, J., Bravar, U., Kanbach, G., Andritschke, R., Ajello, M., Zoglauer, A., Hunter, S., Phlips, B., Wulf, E., Hartmann, D., Miller, R., Paciesas, W., Zych, A., Kippen, R., Vestrand, T., Cherry, M., Guzik, T., Stacy, J., Wefel, J., Reglero, V., Di Cocco, G., \& Cravens, J. (2005). MEGA: A Medium-Energy Gamma-ray Astronomy mission concept. Proceedings of SPIE - The International Society for Optical Engineering, 5898, 1-12. https://doi.org/10.1117/12.617315

This Conference Proceeding is brought to you for free and open access by the Department of Physics \& Astronomy at LSU Digital Commons. It has been accepted for inclusion in Faculty Publications by an authorized administrator of LSU Digital Commons. For more information, please contact ir@lsu.edu. 


\section{Authors}

P. F. Bloser, J. M. Ryan, M. L. McConnell, J. R. Macri, U. Bravar, G. Kanbach, R. Andritschke, M. Ajello, A. Zoglauer, S. D. Hunter, B. F. Phlips, E. A. Wulf, D. H. Hartmann, R. S. Miller, W. S. Paciesas, A. D. Zych, R. M. Kippen, T. Vestrand, M. L. Cherry, T. G. Guzik, J. G. Stacy, J. P. Wefel, V. Reglero, G. Di Cocco, and J. P. Cravens 
University of New Hampshire

University of New Hampshire Scholars' Repository

Space Science Center

Institute for the Study of Earth, Oceans, and

Space (EOS)

$9-2-2005$

\title{
MEGA: a medium-energy gamma-ray astronomy mission concept
}

\author{
Peter F. Bloser \\ University of New Hampshire, Peter.Bloser@unh.edu \\ James M. Ryan \\ University of New Hampshire, James.Ryan@unh.edu \\ Mark L. McConnell \\ University of New Hampshire - Main Campus, mark.mcconnell@unh.edu \\ John R. Macri \\ University of New Hampshire - Main Campus, John.Macri@unh.edu \\ U Bravar \\ University of New Hampshire - Main Campus
}

See next page for additional authors

Follow this and additional works at: https://scholars.unh.edu/ssc

Part of the Astrophysics and Astronomy Commons

\section{Recommended Citation}

P. F. Bloser ; J. M. Ryan ; M. L. McConnell ; J. R. Macri ; U. Bravar ; G. Kanbach ; R. Andritschke ; M. Ajello ; A. Zoglauer ; S. D. Hunter ; B. F. Phlips ; E. A. Wulf ; D. H. Hartmann ; R. S. Miller ; W. S. Paciesas ; A. D. Zych ; R. M. Kippen ; T. Vestrand ; M. L. Cherry ; T. G. Guzik ; J. G. Stacy ; J. P. Wefel ; V. Reglero ; G. Di Cocco and J. P. Cravens "MEGA: a medium-energy gamma-ray astronomy mission concept", Proc. SPIE 5898, UV, XRay, and Gamma-Ray Space Instrumentation for Astronomy XIV, 589804 (September 02, 2005); doi:10.1117/12.617315; http://dx.doi.org/10.1117/12.617315

This Conference Proceeding is brought to you for free and open access by the Institute for the Study of Earth, Oceans, and Space (EOS) at University of New Hampshire Scholars' Repository. It has been accepted for inclusion in Space Science Center by an authorized administrator of University of New Hampshire Scholars' Repository. For more information, please contact Scholarly.Communication@unh.edu. 


\section{Authors}

Peter F. Bloser, James M. Ryan, Mark L. McConnell, John R. Macri, U Bravar, G Kanbach, R Andritsche, M Ajello, A Zoglauer, S Hunter, B F. Phlips, Eric Wulf, Dieter H. Hartman, R S. Miller, W Paciesas, Allen Zych, R M. Kippen, W T. Vestrand, M L. Cherry, T G. Guzik, J G. Stacy, J P. Wefel, V Reglero, G DiCocco, and J Cravens 


\title{
MEGA: A Medium-Energy Gamma-ray Astronomy Mission Concept
}

\author{
P. F. Bloser ${ }^{a}$, J. M. Ryan ${ }^{a}$, M. L. McConnell ${ }^{a}$, J. R. Macri ${ }^{a}$, U. Bravar $^{a}$, G. $\operatorname{Kanbach}^{b}$, R. \\ Andritschke $^{b}$, M. Ajello ${ }^{b}$, A. Zoglauer ${ }^{c}$, S. D. Hunter ${ }^{d}$, B. F. Phlips ${ }^{e}$, E. A. Wulf ${ }^{e}$, D. H.

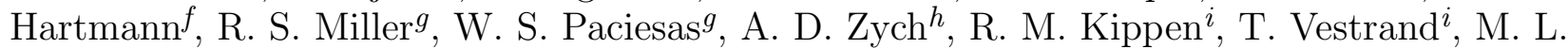 \\ Cherry $^{j}$, T. G. Guzik ${ }^{j}$, J. G. Stacy ${ }^{j}$, J. P. Wefel ${ }^{j}$, V. Reglero ${ }^{k}$, G. Di Cocco ${ }^{l}$, and J. P. \\ Cravens $^{m}$ \\ ${ }^{a}$ Space Science Center, University of New Hampshire, Durham, NH, USA; \\ ${ }^{b}$ Max-Planck-Institut für extraterrestrische Physik, Garching, Germany; \\ ${ }^{c}$ Space Sciences Laboratory, University of California, Berkelely, CA, USA; \\ ${ }^{d}$ NASA Goddard Space Flight Center, Code 661, Greenbelt, MD, USA; \\ ${ }^{e}$ Naval Research Laboratory, Washington, DC, USA; \\ ${ }^{f}$ Physics Department, Clemson University, Clemson, SC, USA; \\ ${ }^{g}$ Physics Department, University of Alabama, Huntsville, AL, USA; \\ ${ }^{h}$ Institute of Geophysics \& Planetary Physics, University of California, Riverside, CA, USA; \\ ${ }^{i}$ Los Alamos National Laboratory, Los Alamos, NM, USA; \\ ${ }^{j}$ Physics Department, Louisiana State University, Baton Rouge, LA, USA; \\ ${ }^{k}$ Grupo de Astronomía y Ciencias del Espacio, University of Valencia, Valencia, Spain; \\ ${ }^{l}$ Istituto di Astrofisica Spaziale e Fisica Cosmica, Bologna, Italy; \\ ${ }^{m}$ Southwest Research Institute, San Antonio, TX, USA
}

\begin{abstract}
The Medium Energy Gamma-ray Astronomy (MEGA) telescope concept will soon be proposed as a MIDEX mission. This mission would enable a sensitive all-sky survey of the medium-energy gamma-ray sky $(0.4-50$ $\mathrm{MeV}$ ) and bridge the huge sensitivity gap between the COMPTEL and OSSE experiments on the Compton Gamma Ray Observatory, the SPI and IBIS instruments on INTEGRAL, and the visionary Advanced Compton Telescope (ACT) mission. The scientific goals include, among other things, compiling a much larger catalog of sources in this energy range, performing far deeper searches for supernovae, better measuring the galactic continuum and line emissions, and identifying the components of the cosmic diffuse gamma-ray emission. MEGA will accomplish these goals using a tracker made of Si strip detector (SSD) planes surrounded by a dense high$\mathrm{Z}$ calorimeter. At lower photon energies (below $\sim 30 \mathrm{MeV}$ ), the design is sensitive to Compton interactions, with the SSD system serving as a scattering medium that also detects and measures the Compton recoil energy deposit. If the energy of the recoil electron is sufficiently high $(>2 \mathrm{MeV})$ its momentum vector can also be measured. At higher photon energies (above $\sim 10 \mathrm{MeV}$ ), the design is sensitive to pair production events, with the SSD system measuring the tracks of the electron and positron. A prototype instrument has been developed and calibrated in the laboratory and at a gamma-ray beam facility. We present calibration results from the prototype and describe the proposed satellite mission.
\end{abstract}

Keywords: gamma rays, astrophysics, Compton telescopes, satellite mission, silicon strip detectors, scintillators

Further author information: (Send correspondence to P. F. Bloser)

P. F. Bloser: E-mail: Peter.Bloser@unh.edu

UV, X-Ray, and Gamma-Ray Space Instrumentation for Astronomy XIV edited by Oswald H. W. Siegmund, Proc. of SPIE Vol. 5898 589804, (2005) - 0277-786X/05/\$15 - doi: 10.1117/12.617315

Proc. of SPIE Vol. 5898 589804-1 


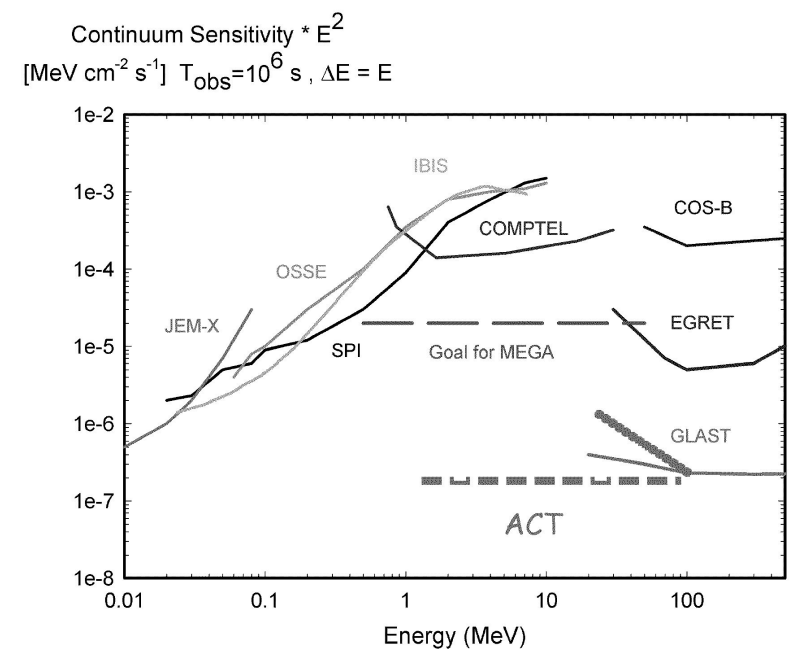

Figure 1. Sensitivity curves for recent and current gamma-ray and X-ray telescopes, showing the sensitivity goal for a MEGA satellite mission.

\section{INTRODUCTION}

In the field of gamma-ray astronomy there is an urgent need for a new mission in the medium-energy $(\sim 0.4-50$ $\mathrm{MeV}$ ) range to follow up on the success of the COMPTEL telescope on the Compton Gamma-Ray Observatory. ${ }^{1}$ This energy band is crucial for the study of a rich variety of high-energy astrophysical processes. The goal of the Medium Energy Gamma-ray Astronomy (MEGA) project is to meet this need, using modern detector technology to improve on the sensitivity of COMPTEL by a factor of ten.

Astronomy with medium-energy gamma rays has always been an experimental challenge. There are two main reasons for this: (1) Photon interaction cross-sections in the $\mathrm{MeV}$ range go through a minimum from the absence of the photoelectric effect $(\sim 100 \mathrm{keV})$ and pair production $(\sim 10 \mathrm{MeV})$. In this range Compton scattering is the dominant process, and so the few interactions are characterized by small energy deposits and long-range secondary radiation. It is therefore necessary to build a "deep" detector to achieve a reasonable efficiency and to finely segment the detector to record and trigger widely separated interactions. (2) The nuclear energy levels of all detector and structural materials lie in the $\mathrm{MeV}$ range and are easily excited by energetic particles. The result of this systematic "radio-activation" is an intense local gamma-ray background that must be re-jected to achieve the required sensitivity.

COMPTEL was the first instrument in space to explore this difficult spectral band with a practical sensitivity. COMPTEL required that an incident photon Compton scatter in a liquid scintillator detector (D1) and be subsequently photo-absorbed in a thick NaI detector (D2) located $1.6 \mathrm{~m}$ below. A time of flight (ToF) measurement ensured that only events fitting this sequence were accepted. The effective area of COMPTEL was limited by poor absorption and the small solid angle for scattering. The sensitivity was further reduced by poor angular resolution and high background.

The need for new mission in medium-energy gamma-ray astronomy is illustrated in Fig. 1, which shows the point source sensitivity of COMPTEL compared to that of past, present, and future instruments in neighboring energy bands. The "sensitivity gap" from $0.5-50 \mathrm{MeV}$ is evident. In contrast, above $100 \mathrm{MeV}$ development has proceeded through three successive generations of instrumentation (COS-B, EGRET, and now GLAST, scheduled to launch in 2007), each achieving about a $10 \times$ improvement in sensitivity. A similar path of development is needed in the medium-energy band. The 2003 NASA Structure and Evolution of the Universe Roadmap calls for a mission known as the Advanced Compton Telescope (ACT) to achieve a factor of $\sim 100$ better sensitivity than that of COMPTEL. This mission is currently the subject of a NASA Vision Mission Concept Study. This 
Concept Study is critically evaluating advanced detector technologies and developing sophisticated Monte Carlo simulation tools to determine how ACT can be designed. It is already clear that the technical challenges are formidable. In terms of the higher energy instruments, developing ACT now is the equivalent of going directly from COS-B to GLAST. An intermediate step is required that MEGA is designed to fulfill. A sky survey at a sensitivity that repeats what EGRET provided at $100 \mathrm{MeV}$ will identify new objects that will be targets of ACT. At the same time, MeV objects that are or were merely detected with COMPTEL or INTEGRAL can now be studied quantitatively and in some detail. This is the goal of the MEGA project. ${ }^{2}$

\section{SCIENTIFIC MOTIVATION}

A broad range of astrophysical science is best studied via observations of the Universe in the medium-energy gamma-ray band. Medium-energy gamma rays probe extreme physical conditions in the Universe that give rise to nuclear interactions and relativistic particles. The physics at work in these locations determines how stars die and new elements are born. Investigating this radiation will help us understand the behavior of black holes and the fate of the Universe. The only instrument in this energy range to fly in space with sufficient sensitivity to make useful astronomical observations was the COMPTEL instrument on the CGRO satellite. COMPTEL provided only a dim, blurry view of the gamma-ray Universe, but it was sufficient to demonstrate that the potential science return of a more sensitive instrument will be rich indeed. Specifically, a significantly more sensitive instrument would address the following:

- Search for and measurement of supernova emissions to better understand the dynamics of the explosion process and the creation of the elements. The anomalous redshift velocities of distant Type Ia supernovae have led to a critical review of the value of the cosmological constant. It is important to fully understand the Type Ia process since it is used here as a standard candle to evaluate the Hubble constant. COMPTEL was able to place upper limits on ${ }^{56}$ Co gamma-ray line emission from one supernova and marginally measure another. ${ }^{3}$ With a $10 \times$ improvement in sensitivity, it is likely that a Type Ia supernova will occur in a five-year period within a radius where good measurements can be conducted so as to test our models of the phenomenon.

- Measurements of radioactive decay line emission from supernova remnants. Improving the COMPTEL ${ }^{44} \mathrm{Ti}$ luminosity measurements of Cas $\mathrm{A}^{4}$ would be accompanied by luminosity measurements of ${ }^{26} \mathrm{Al}$ and ${ }^{60} \mathrm{Fe}$. These isotopes drive the last phases of the supernova expansion and populate the Galaxy with radioactive ash.

- The isotope ${ }^{26} \mathrm{Al}$ populates the plane of the Galaxy producing a diffuse Galactic emission. It was mapped by COMPTEL, ${ }^{5}$ but higher definition versions of these maps are necessary to properly associate the emission with distinct galactic structures and their progenitor stars.

- The origin of the diffuse Galactic continuum emission at $\mathrm{MeV}$ energies is still very mysterious: current models of galactic gamma-ray production in cosmic ray-gas interactions fail to provide a satisfactory explanation for this galactic glow. ${ }^{6}$

- Black hole candidates and AGNs exhibit emission in the MeV range, probably from the re-energization of $\mathrm{X}$ rays by scattering off high-energy electrons. The $\mathrm{MeV}$ tail on the spectrum of $\mathrm{Cyg} \mathrm{X}-1^{7}$ represents the kind of exciting physics that can emerge when studying such objects. AGNs have been associated with emission above $100 \mathrm{MeV}$, but this is likely due to the limited sensitivity that exists in the MeV band. It is likely that a broader picture of AGNs will emerge when more are measured in the $\mathrm{MeV}$ band. The object $3 \mathrm{C} 273$ is such an example of an AGN that is luminous in $\mathrm{MeV}$ gamma rays.

- Classical novae produce other isotopes that radiate in the $\mathrm{MeV}$ range that again will constrain the dynamics of the origin of lighter elements.

- Pulsars should be common in the Galaxy if one has the sensitivity to detect them. COMPTEL detected a handful, all displaying different behaviors in spectrum, light curve, and frequency. This diversity makes categorizing them difficult. A larger set of gamma-ray pulsars might exhibit trends that could be used to understand them. 
- Gamma-ray bursts exhibit some of their most interesting features at energies higher than the classical 100$200 \mathrm{keV}$. They have persisted for time scales of an hour at $\mathrm{GeV}$ energies, ${ }^{8}$ but studies of their long-term behavior at $\mathrm{MeV}$ energies have been limited by sensitivity.

- The spectrum of the cosmic diffuse emission appears from research on COMPTEL, EGRET and SMM data to be comprised of several components. These components include active galaxies and supernovae. The supernova component is the least resolved and understood. A new mission would greatly improve on the COMPTEL measurements of this spectrum from below $1 \mathrm{MeV}$ to $50 \mathrm{MeV}$ and help establish the magnitudes of the different contributions.

- Solar flares exhibit the most complex spectra in the sky. The richness of nuclear lines, bremsstrahlung emission, positron annihilation, and neutron capture contains much information about the processes of ion acceleration that produce these lines. Because of the complexity of the spectrum, the diagonal instrument response of a Compton telescope will greatly aid in the interpretation of the data.

- Many of the phenomena described above probably produce gamma radiation that is at least partially polarized. A gamma-ray telescope based on Compton scattering is in principle sensitive to polarization and thus offers a new diagnostic tool for the study of high-energy astrophysical processes. ${ }^{9}$

A new mission must not only have greater effective area to accept and register more gamma-rays, but it must retain or improve background rejection. To attain a larger effective area than COMPTEL does not necessarily imply a physically larger or more massive experiment. By bringing the two detectors closer together, the solid angle factor can be markedly increased. However, moving these detectors closer together comes at a cost: at the small distances necessary, ToF discrimination becomes problematic. Another method for rejecting background is necessary. Below we describe how MEGA provides a dramatic improvement in effective area while retaining the background rejection power that COMPTEL enjoyed by tracking the Compton recoil electron. This ability makes MEGA sensitive to pair production events as well.

\section{THE MEGA CONCEPT AND PROTOTYPE}

\subsection{Principle of Operation}

Two physical processes dominate the interaction of photons with matter in the medium-energy gamma-ray band: Compton scattering at low energies, and electron-positron pair production at high energies, with the changeover near $10 \mathrm{MeV}$ for most detector materials. In both cases the primary interaction produces long-range secondaries whose momenta must be measured.

If one could trace the Compton scattering within an instrument then the incident direction and energy of the gamma ray could be determined through the Compton formula

$$
\cos \phi=1+\frac{m_{e} c^{2}}{E_{\gamma}}-\frac{m_{e} c^{2}}{E_{\gamma}-E_{1}}
$$

where $\phi$ is the scatter angle of the photon, $E_{\gamma}$ is the photon energy and $E_{1}$ is the energy transferred to the recoil electron. With a complete measure of $E_{\gamma}$ and a measure of the recoil-electron momentum vector, one has the complete information about the incident gamma ray. Without this one can only constrain the photon energy or its incident direction. Without measuring the recoil-electron direction the incident direction is restricted to the mantle of a cone whose axis is the direction of the scattered gamma ray (see Fig. 2).

MEGA, like previous Compton and pair creation telescopes, employs two independent detectors: a tracker (D1) made of double-sided Si strip detectors, in which the initial Compton scatter or pair conversion takes place, and a calorimeter (D2) made of pixellated CsI detectors, that absorb and measure the energy of the secondaries (see Fig. 2). For Compton interactions, the incident photon scatters off an electron in the tracker. The interaction position and the energy imparted to the electron are measured. The scattered photon interaction point and energy are recorded in the calorimeter. From the positions and energies of the two interactions the incident photon angle $\phi$ is computed from the Compton equation, Eq. 1. The primary-photon incident direction 


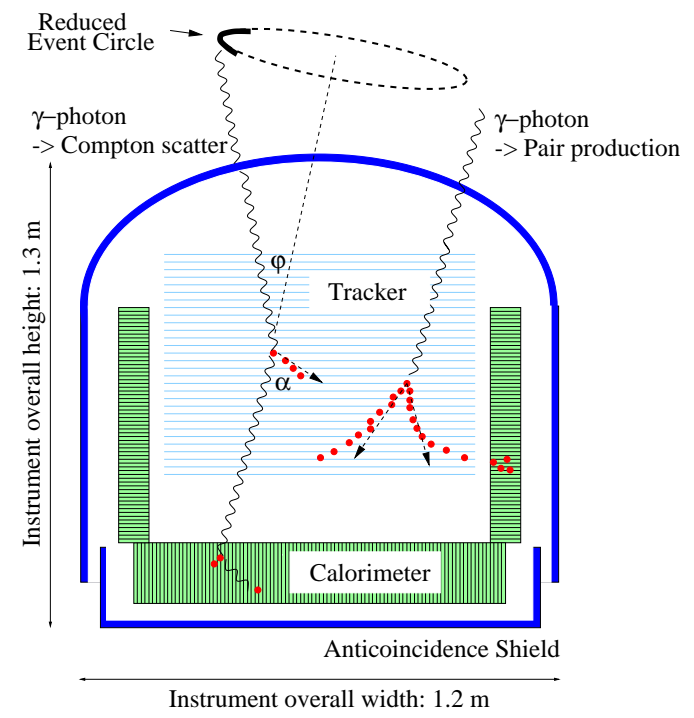

Figure 2. Schematic of the MEGA telescope showing the kinematics of gamma-ray interactions.

is then constrained to an event circle on the sky. For incident energies above about $2 \mathrm{MeV}$ the recoil electron usually receives enough energy to penetrate several Si layers, allowing it to be tracked. This further constrains the incident direction of the photon to an arc (the reduced event circle in Fig. 2). In the case of pair production, the incident photon converts into an electron-positron pair in the tracker. These two particles are tracked and determine the incident photon direction. The total energy is then measured through the deposits in the tracker and/or the calorimeter.

The differential Klein-Nishina cross section for Compton scattering contains a strong dependence on the polarization of the incident gamma-ray photon. Scattered photons are emitted preferentially perpendicular to the direction of the electric field vector of the incoming photon. The strongest azimuthal modulation in the distribution of detected photons will be for gamma-ray energies between 1-5 MeV and scatter angles of $20^{\circ}-60^{\circ}$. This makes a Compton telescope with a calorimeter covering a large solid angle a unique polarimeter for gamma radiation.

The design of a new gamma-ray telescope must be based on numerical simulations as well as experimental detector developments. From the concept in Fig. 2 we have used the CERN Monte Carlo package GEANT3 to develop the baseline for a satellite version of MEGA. In this version, the tracker contains 32 layers, while the calorimeter is $8 \mathrm{~cm}$ deep on the bottom and $4 \mathrm{~cm}$ deep on the sides. These choices motivated the decision to construct a representative prototype detector (Fig. 3) of about $25 \%$ of the area and $33 \%$ of the depth of the full satellite version. This prototype has been used to evaluate the performance characteristics and validate the simulations.

\subsection{Components of the Prototype}

Tracker: The tracker must accomplish several tasks. It must: (1) act as the scattering medium for Compton interactions; (2) measure the scatter location and the energy imparted to the recoil electron; (3) act as the conversion medium for pair production; (4) provide a large interaction volume for both processes; (5) record the tracks and energy deposits of all secondary particles, both electron-positron pairs and Compton recoil electrons; (6) provide a fast timing signal to be used in a coincident trigger with the calorimeter; and (7) operate without elaborate cooling and with reasonable power consumption. Thus, good position, energy, and time resolution are required simultaneously in a large volume. The logical choice of detector technology for the tracker was a stack of double-sided silicon strip detectors. ${ }^{10}$ These detectors were designed by the MPE semiconductor laboratory and produced by Eurisys Mesures, Lingolsheim, France. Each tracker layer is composed of a $3 \times 3$ array of $500 \mu \mathrm{m}$ thick silicon wafers, each $6 \times 6 \mathrm{~cm}^{2}$ in size and fitted with 128 orthogonal $\mathrm{p}$ and $\mathrm{n}$ strips on opposite 


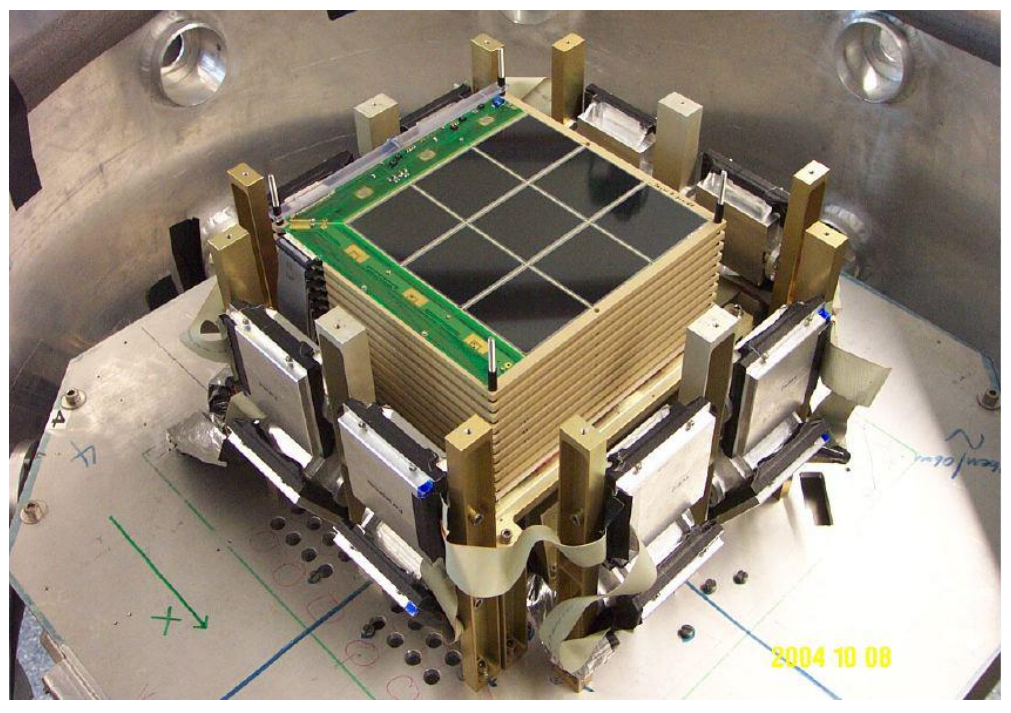

Figure 3. The MEGA prototype including Si strip detectors (D1) and CsI detectors (D2).

sides (470 $\mu \mathrm{m}$ pitch). The strips are biased using the punch-through principal and AC-coupled via metal strips separated from the strip implant by an insulating oxide/nitride layer. The strips from adjacent wafers in the $3 \times 3$ array are wire-bonded in series and read out by 128-channel TA1.1 ASICs, creating a total area of $18 \times 18$ $\mathrm{cm}^{2}$ position-sensitive area. At $20^{\circ} \mathrm{C}$ a typical energy resolution of $15-20 \mathrm{keV}$ FWHM, a position resolution of $290 \mu \mathrm{m}$ (measured with muon tracks), and a time resolution of $\sim 1 \mu \mathrm{s}$ is measured. The stack in the MEGA prototype detector (Fig. 3) contains 11 layers. Fig. 3 also shows the front-end electronics boards mounted next to the Si wafers. The tracker for the satellite size MEGA could be assembled from four quadrants, each similar to the prototype tracker, with the electronics on the outer rim.

Calorimeter: The MEGA prototype calorimeter ${ }^{11}$ consists of 20 modules, each with an array of $10 \times 12$ CsI(Tl) scintillator bars of cross-section $5 \times 5 \mathrm{~mm}^{2}$ read out with silicon PIN-diodes and the same TA1.1 ASICs as the tracker. The length of the bars (the depth of the calorimeter) was chosen to correspond to the stopping power needed to absorb the scattered photons at different angles; i.e., large scatter angles produce low energy secondary photons that are stopped in thinner crystals while forward scattering requires a thicker calorimeter. The upper side modules are $2 \mathrm{~cm}$ deep, the lower side wall $4 \mathrm{~cm}$, and the bottom calorimeter is $8 \mathrm{~cm}$ deep. The CsI(Tl) bars were manufactured by Hilger Crystals and are polished on all sides. The bars are packed into the modules with optical separation provided by several layers of reflective paper (Millipore). The monolithic PIN diode arrays were manufactured by Hamamatsu and are coupled to the scintillators with a prefabricated layer of transparent silicone cushions where the individual pixels are separated by a grid of opaque white silicon. The $8 \mathrm{~cm}$ modules are read out from both ends. The ratio of the two signals allows the localization of the energy deposit along the bar with a typical resolution of $\sim 2 \mathrm{~cm}$ (FWHM). This 3-D resolution, together with the magnitude of the energy deposit, is important for reconstructing the correct sequence of multiple interactions in the calorimeter. The energy resolution of the calorimeter modules was measured with radioactive sources. Typical results for $\triangle \mathrm{E} / \mathrm{E}$ (FWHM) at the incident energy of $662 \mathrm{keV}$ range between $10 \%$ (for a $2 \mathrm{~cm}$ unit) and $14 \%$ (for $8 \mathrm{~cm}$ ). The energy resolution of the calorimeter is now the limiting factor for the angular resolution of the MEGA design (see Section 3.3.1).

Electronics, Trigger, and Data Acquisition: The MEGA prototype contains 8448 and 2880 readout channels in the tracker and calorimeter respectively. A satellite telescope with the same pixellation will contain more than $10 \times$ this number. The data acquisition demands highly integrated, low power electronics. For the prototype instrument the front-end, repeater card (see below), opto-coupler, trigger processing card, and power supplies for electronics and detectors were custom developments at MPE. The back-end, with the signal transceivers, sequencer card, analog-to-digital converters (ADCs), and an on-board computer (OBC) with mass 
storage (hard disk), is based on VME modules. The data acquisition and detector control software is based on $\mathrm{C}++$ and the ROOT software library from CERN. It operates on the PC Linux system (Pentium III, $850 \mathrm{MHz}$ ) of the OBC.

The front-ends of both the tracker and calorimeter use VLSI amplifier-ASICs of type TA1.1 designed by Ideas ASA (Norway) with built-in trigger capability. This ASIC has the following characteristics: (1) low noise level of $165 \mathrm{e}^{-}+7 \mathrm{e}^{-} / \mathrm{pF}$ with an adjustable shaping time $(2 \mu$ s for MEGA); (2) a dynamic range of \pm 5 MIPs; (3) 128 channels in parallel with serial readout up to a $10 \mathrm{MHz}$ clock rate; (4) low power consumption of $\sim 1.5$ $\mathrm{mW} /$ channel (5) common trigger output from 128 level discriminators with common adjustable threshold; and (6) a 128-bit blocking register to prevent noisy channels from triggering.

A so-called repeater card supplies 3 ASICs with low-noise power, shifts and amplifies signals, and generates the analog trigger threshold levels. All signals pass through opto-couplers to galvanically isolate the front ends, which operate at different bias potentials, from one another and the rest of the system. The trigger signals from all chips are routed to the trigger-processing unit. An absolute veto and a programmable veto (from an anticoincidence shield, for example) are possible via inputs to the trigger card. If no veto is received, the trigger pattern, in the form of a 21-bit word, is used as an address to interrogate a 2 Mbit RAM where the valid trigger combinations are stored as non-zero bits. The flexibility to generate different memory loads for this trigger register allows one to select the best events and to adjust the trigger rate to an acceptable level. The trigger processor is implemented on an FPGA (Xilinx-Spartan) and is housed in a slot of the VME back-end. With a valid trigger pattern the trigger card sends a hold signal and the sequencer carries out the readout, addressing each channel (up to 8 in parallel) for digitization by the ADCs. The data for each event are stored in the OBC storage medium.

\subsection{Prototype Performance and Calibration}

From January to March 2003 the prototype was calibrated with laboratory sources $\left({ }^{22} \mathrm{Na},{ }^{137} \mathrm{Cs},{ }^{88} \mathrm{Y}\right)$ in the near field. In April/May 2003 it was calibrated at the High Intensity Gamma Source ${ }^{12}(\mathrm{HI} \gamma \mathrm{S})$ at Duke University (Durham, North Carolina) in the far field. ${ }^{13-15}$ The latter calibration used mono-energetic $(\Delta \mathrm{E} / \mathrm{E}<2 \%)$ and $100 \%$ polarized pencil beams at different energies $(0.7,2,5,8,10,12,17,25,37$ and $49 \mathrm{MeV})$ and different incident angles $\left(0^{\circ}, 30^{\circ}, 60^{\circ}, 80^{\circ}, 120^{\circ}, 180^{\circ}\right)$. Based on these data, preliminary imaging properties of the telescope were determined. These are the most thorough calibrations of a prototype advanced Compton telescope ever performed.

The image reconstruction is performed using an unbinned maximum-likelihood method called list-mode maximum-likelihood expectation-maximization, which originally was developed for medical imaging of a SPECT camera ${ }^{16}$ and later adapted for astrophysics. ${ }^{17,18}$ This method uses different event types (tracked and un-tracked Compton events as well as pair events) in one image while preserving all measured information.

\subsubsection{Compton Scatter Regime}

Ideally, an electron-tracking Compton telescope should measure all parameters to directly calculate the origin of the incoming photon: the direction and energy of the scattered photon and the direction and energy of the recoil electron. Below $2 \mathrm{MeV}$ the energy of the electron is in most cases not sufficient to generate a track in D1. For these events the origin of the photon can only be restricted to the classical cone section. The angular width of this cone mantle is mainly determined by the energy resolution of the detectors. Even with knowledge of the electron track, Molière (small-angle) scattering in the silicon produces an uncertainty in the direction of the electron. The angular width of the cone mantle in combination with the electron track restricts the possible origins of the photon to short arcs (the reduced event circle in Fig. 2).

Angular Resolution: A measure of the width of the event circle or arc is the Angular Resolution Measure (ARM), defined as the difference between the real and the measured Compton scatter angle. An example is given in Fig. $4 .{ }^{15}$ This distribution contains tracked as well as untracked events at $2.0 \pm 0.2 \mathrm{MeV}$ and has a FWHM of $7.4^{\circ}$. If only untracked events are considered, the shape is narrower $\left(6.2^{\circ}\right)$, whereas tracked events produce a much broader ARM $\left(13.4^{\circ}\right)$. This behavior is expected, since tracked events contain relatively more energy in the electron and less in the scattered photon, and so are more sensitive to the limited energy resolution of the calorimeters. The physical limit of the ARM is set by the non-zero, Z-dependent momentum of the scattering 


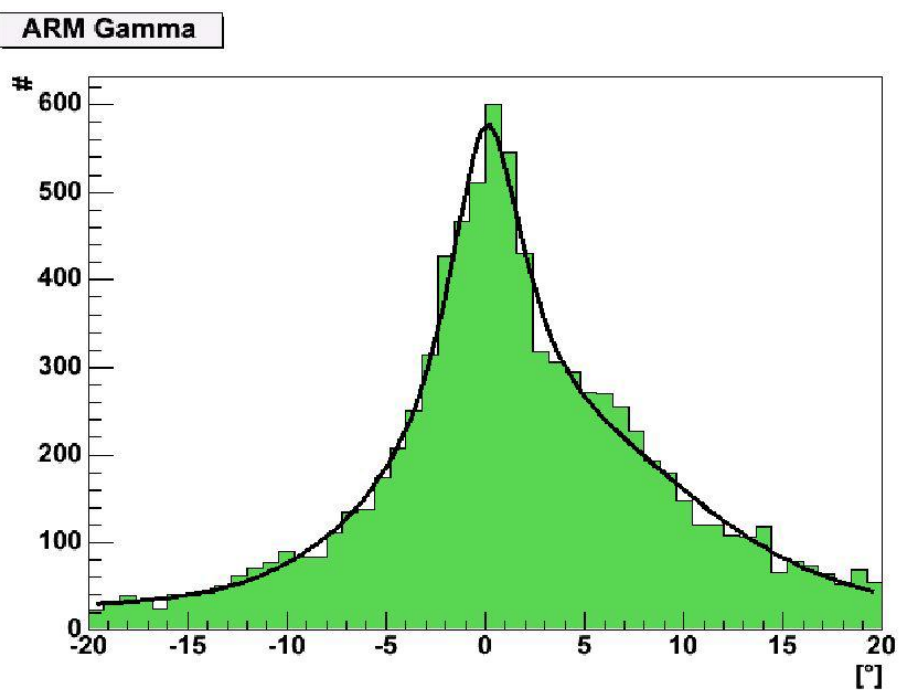

Figure 4. Measured ARM distribution for tracked and untracked events from 1.8 to $2.2 \mathrm{MeV}$ with a $\mathrm{FWHM}$ of $\sim 7.4^{\circ}$.

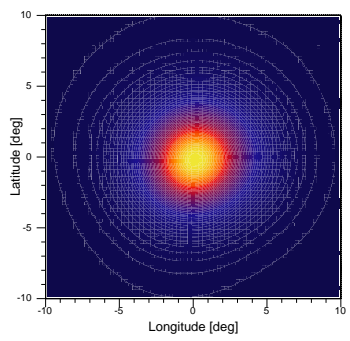

$710 \mathrm{keV}$

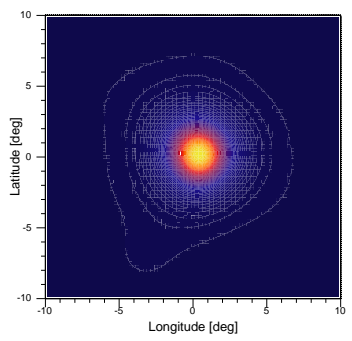

$2 \mathrm{MeV}$

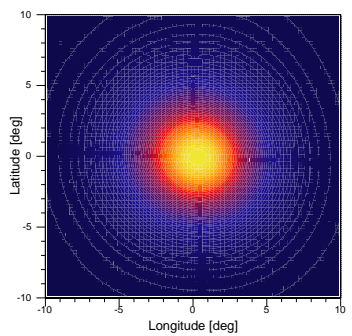

$5 \mathrm{MeV}$

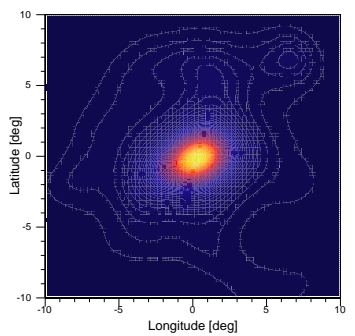

$8 \mathrm{MeV}$

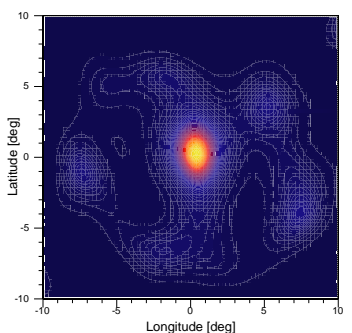

Figure 5. Compton images from the $\mathrm{HI} \gamma \mathrm{S}$ calibration. The angular resolution improves with energy due to the smaller relative errors in the energy measurement. Images are made using either untracked (top) or tracked (bottom) events only. Each image is $20^{\circ}$ across. The angular resolution for tracked events is currently limited by the energy resolution of the calorimeter.

electron, i.e. Doppler-broadening. At $2 \mathrm{MeV}$ Doppler-broadening leads to an average width of $\sim 0.2^{\circ}$ in silicon. ${ }^{14}$ A calorimeter with good energy resolution is therefore important.

The angular resolution at lower energies is mainly determined by the measurement error of the energy. Going from lower to higher energies the performance improves, as shown in Fig. 5 using Compton images from the HI $\gamma \mathrm{S}$ calibration. The worsening of the angular resolution for tracked events at $2 \mathrm{MeV}$ is again evident. It should be noted that, although the angular resolution for tracked events is currently degraded, tracked events still are less dominated by background due to the smaller solid angle of the reduced event circle (Fig. 2). 


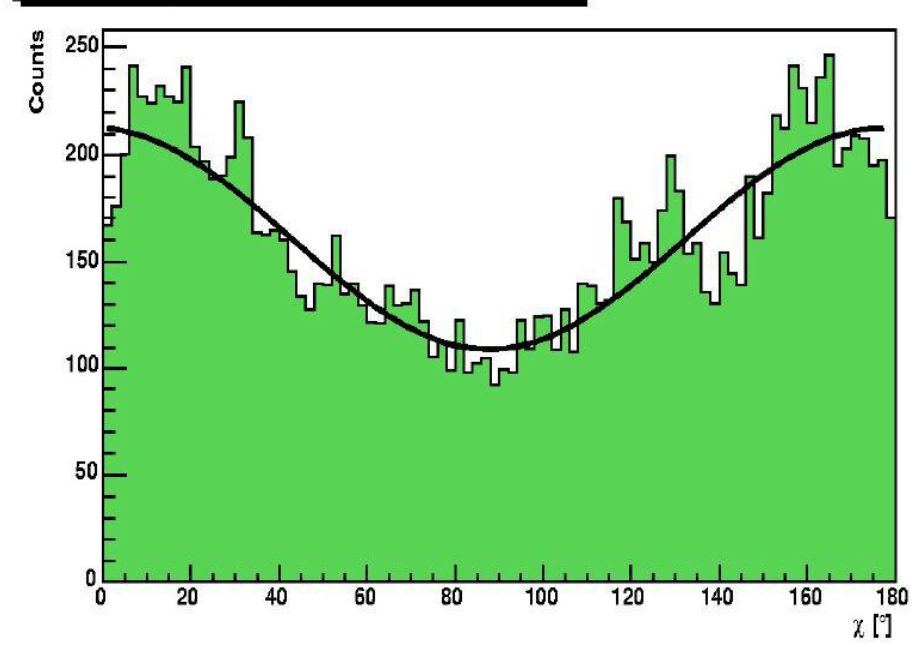

Figure 6. Geometry-corrected distribution of the azimuthal scatter angle for $100 \%$ polarized photons (710 keV).

Direction of Electron Tracks: The most important characteristic of a tracking Compton telescope is the electron track. At $1.8 \mathrm{MeV}\left({ }^{88} \mathrm{Y}\right)$ the HWHM of the track direction error distribution is $\sim 42^{\circ} .{ }^{15}$ This is close to the physical limit determined by Molière scattering. Even at this relatively low energy more than $85 \%$ of all tracks can be reconstructed correctly.

Polarization: Compton telescopes are inherently sensitive to polarization. Fig. 6 shows the azimuthal scatter distribution measured with $100 \%$ polarized $710 \mathrm{keV}$ gamma rays produced at the HI $\gamma \mathrm{S}$ facility at Duke University. ${ }^{15}$ Because MEGA is not axially symmetric, the distribution has been corrected using a measurement with an unpolarized ${ }^{137} \mathrm{Cs}$ source $(662 \mathrm{keV})$ positioned one meter above the detector. The polarization is characterized by the modulation factor $\mu=\left(N_{\max }-N_{\min }\right) /\left(N_{\max }+N_{\min }\right)$, where $N_{\max }$ and $N_{\min }$ are the number of counts at the maximum and the minimum of the azimuth distribution. With the $100 \%$ polarized beam $\mu_{100}=0.31 \pm 0.03$ (Fig. 6). Monte Carlo simulations predict $\mu_{100}=0.304$. The geometry of the instrument/beam setup should produce a maximum at $0^{\circ}$, whereas the maximum was measured to be $-4.6^{\circ} \pm 2.1^{\circ}$. Other measurements at $2 \mathrm{MeV}$ with poorer statistics yield a modulation of $\mu_{100}=0.13 \pm 0.04$, also in agreement with simulations.

Multiple and Extended Sources: In a realistic astrophysical environment a telescope must detect and resolve multiple sources within the field of view. To test the instrument capability to do this we placed five sources of different energies with different intensities at different positions in the field of view of MEGA. All five sources were reconstructed at the correct positions. ${ }^{15}$ It is also necessary to resolve extended sources such as supernova remnants or OB-associations. This ability was demonstrated by mounting two ${ }^{88} \mathrm{Y}$ sources on a rotating propeller located $27 \mathrm{~cm}$ above the center of the tracker. ${ }^{14}$ The reconstructed image is shown in Fig. 7. The sources moved in a circular path of radius $7 \mathrm{~cm}$. This corresponds to an angular diameter of $\sim 29^{\circ}$ at infinity. The image contains 138,000 Compton events in the energy range from 0.8 to $1.0 \mathrm{MeV}$. The non-uniformities in the image are artifacts of the instrument response, an effect that should be correctable.

\subsubsection{Pair-Production Regime}

Angular Resolution: Molière scattering in the Si strip detectors dominates the angular resolution for pair events. The influence of this effect is greatest at threshold $(8 \mathrm{MeV})$ and diminishes at higher energies. The angular resolution, given as the cone half-angle which contains $68 \%$ of all events, is shown in Fig. $8 .{ }^{15}$ It varies from $\sim 18^{\circ}$ at $10 \mathrm{MeV}$ to $<5^{\circ}$ at $49 \mathrm{MeV}$. At $49 \mathrm{MeV}$ this is roughly $2 \times$ better than the EGRET telescope. At energies below $100 \mathrm{MeV}$ it will also surpass the expected performance of GLAST and AGILE, since these telescopes contain thick passive converter foils that scatter the electrons. 


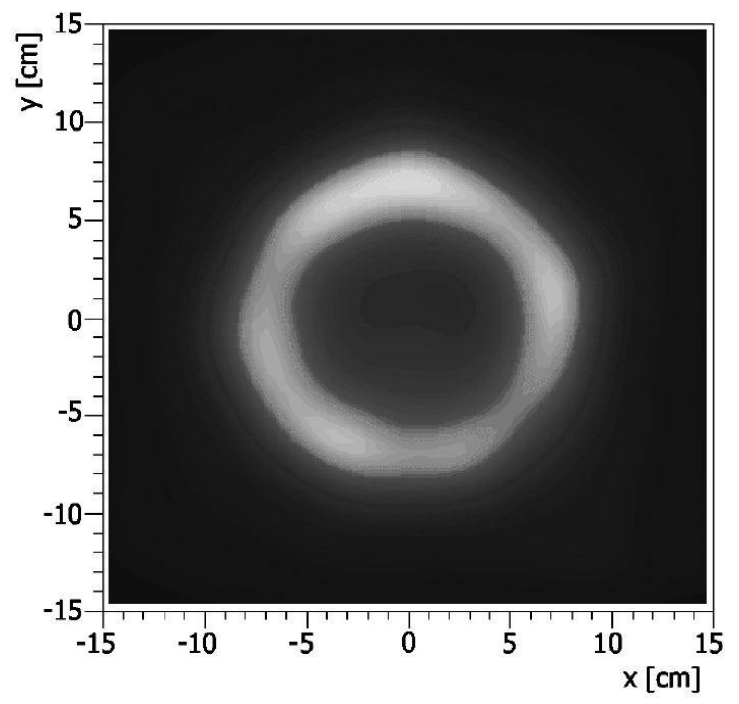

Figure 7. The time-averaged image of two gamma-ray sources moving in a circular path in the field of view.

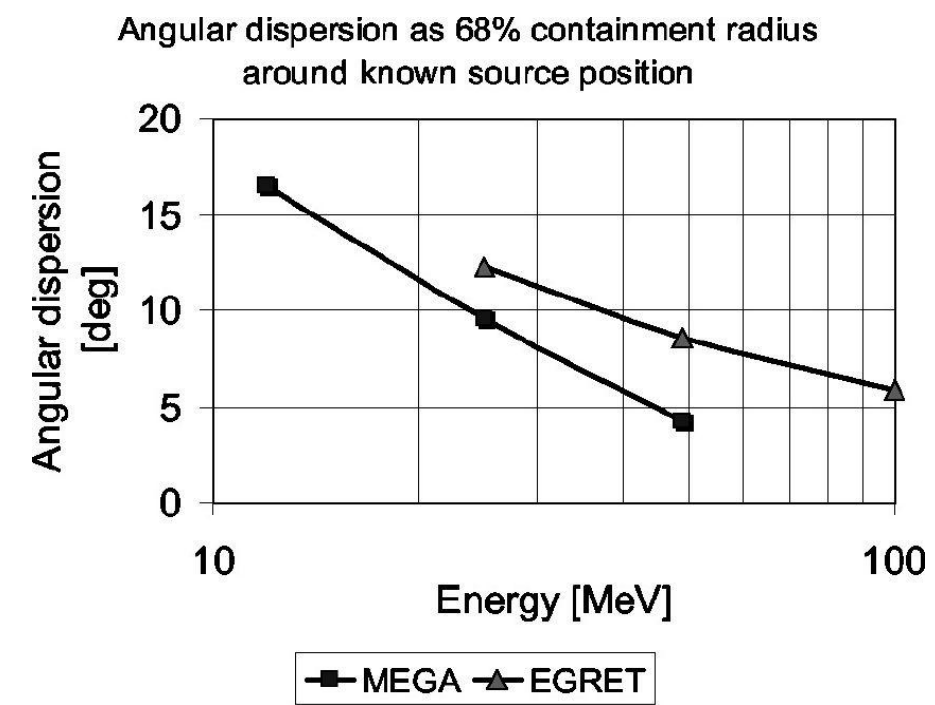

Figure 8. Angular resolution curves for pair-production events for MEGA and EGRET. 


\subsubsection{Calibration Summary}

The Duke calibration demonstrated that the MEGA technique of detecting gamma rays works for a large energy range $(500 \mathrm{keV}$ to $49 \mathrm{MeV}$ ) over a wide field of view. It also showed that MEGA can detect polarization up to at least $2 \mathrm{MeV}$, and that in the pair-production regime the telescope has excellent angular resolution. Poorer angular resolution exists in the Compton regime, because it is dominated by the poor energy resolution of the calorimeter.

\subsection{Future Plans for the MEGA Prototype}

We are currently planning additional laboratory tests with the MEGA prototype to evaluate alternative calorimeter detectors and to validate the background simulation tools being developed as part of the ACT Concept Study. These tests will greatly aid in the design of a MEGA MIDEX mission.

As discussed in Section 3.3.1, the angular resolution of the MEGA telescope for Compton images is limited by the energy resolution of the calorimeter. Several alternative detector technologies are under consideration to improve the calorimeter energy resolution by at least a factor of two. The first test will be to replace the PIN diodes coupled to the CsI crystals with silicon drift diodes (SDDs) currently under development at IASF in Bologna, Italy. ${ }^{19}$ SDD detectors have a low noise level due to the low device output capacitance. A prototype $\mathrm{SDD} / \mathrm{CsI}$ module has been found to have an energy resolution of 5.5\% FWHM at $662 \mathrm{keV}$. Further improvement may be possible with even more advanced detectors. The University of New Hampshire has a program to develop CZT detectors with single-sided strip readout ${ }^{20}$; these have achieved an energy resolution of $\sim 1.5 \%$ FWHM at $662 \mathrm{keV}$. We will also investigate $\mathrm{LaBr}_{3}$, a high-Z scintillator material with far better energy resolution than CsI $\left(<3 \% \mathrm{FWHM}\right.$ at $\left.662 \mathrm{keV}^{21}\right)$ and a very fast timing response.

Additional tests are planned to validate the best available background simulation tools. These include exposing the MEGA prototype to energetic neutrons $(5-50 \mathrm{MeV})$ at the Crocker Nuclear Laboratory at the University of California at Davis to test the predicted activation, and imaging laboratory gamma sources in the presence of a bright "background" source in order to investigate the effects of random coincidences on event reconstruction algorithms.

\section{THE MEGA MISSION}

A mission built around a Compton telescope like MEGA would fit within the NASA MIDEX envelope in terms of mass, power, and cost. The ideal orbit for the MEGA mission should be a variation of a zenith pointing equatorial orbit. The advantage of such an orbit is that the spacecraft and instrument avoid the South Atlantic Anomaly, minimizing the activation in and around the instrument, while keeping the Earth's atmosphere as far from the instrument aperture as possible. Both internal activation and albedo gamma-rays played major roles in limiting the sensi-tivity of COMPTEL. Except for the celestial poles, the sky will get complete coverage without the complexity of regular attitude control and pointing. Dedicated pointings for bursts and targets of opportunities may still be possible at the expense of complicating spacecraft operations and spacecraft complexity.

The baseline MEGA detector has been developed into a small satellite mission design in a pre-phase A study by Daimler-Chrysler Jena Optronic (DJO). In that design, the detector would have a mass of about $650 \mathrm{~kg}$ and dimensions of $1.3 \mathrm{~m}$ diameter by $1.1 \mathrm{~m}$ length. Placed on a standard small satellite platform the payload would have a launch mass of about $950 \mathrm{~kg}$, a diameter of $2.0 \mathrm{~m}$, and a length of $2.3 \mathrm{~m}$. The electrical power requirement would be $\sim 400 \mathrm{~W}$, and the average telemetry rate about $50 \mathrm{kbit} \mathrm{s}^{-1}$. The development time of MEGA to launch could be 5 years with orbital mission of 3-5 years. A two-year survey mission would re-invigorate a rich field of astrophysics. It would build on the success of the Compton Observatory and the continuing success of INTEGRAL. By viewing the Universe deeper than before, it would bring to the surface exciting new phenomena. What remains is a successful test program and an assessment of the background sensitivity to prepare this instrument concept for a space mission. 


\section{ACKNOWLEDGMENTS}

We would like to acknowledge the institutional support of the University of New Hampshire, the Max-Planck Institute, Goddard Space Flight Center, the University of Alabama, Louisiana State University, the Naval Research Laborarory, IGPP, Clemson University, GACE, and IASF/CNR. We are especially grateful to V. Litvinenko and his team for supporting the MEGA calibrations at the Free Electron Laser Laboratory at Duke University.

\section{REFERENCES}

1. V. Schönfelder, et al., "Instrument description and performance of the imaging gamma-ray telescope COMPTEL aboard the Compton Gamma-Ray Observatory," Astrophysical Journal Supplement Series 86, pp. 657692, 1993.

2. G. Kanbach, et al., "Development and calibration of the tracking Compton/pair telescope MEGA," Nuclear Instruments and Methods in Physics Research A 541, pp. 310-322, 2005.

3. D. J. Morris, et al., "Reassessment of the ${ }^{56}$ Co emission from SN 1991T," in Proceedings of the Fouth Compton Symposium, C. D. Dermer, M. S. Strickman, and J. D. Kurfess, eds., AIP 410, p. 1084, 1998.

4. A. F. Iyudin, et al., "COMPTEL observations of Ti-44 gamma-ray line emission from Cas A," Astronomy and Astrophysics 284, p. L1, 1994.

5. R. Diehl, et al., "COMPTEL observations of galactic ${ }^{26} \mathrm{Al}$ emission," Astronomy and Astrophysics 298, p. 445, 1995.

6. A. Strong, et al., "Diffuse continuum gamma rays from the galaxy," Astrophysical Journal 537, p. 763, 2000.

7. M. L. McConnell, et al., "The soft gamma-ray spectral variability of Cygnus X-1," Astrophysical Journal 572, p. 984, 2002.

8. B. L. Dingus, et al., "EGRET observations of $>30 \mathrm{MeV}$ emission from the brightest bursts detected by BATSE," Astrophysics and Space Science 231, p. 187, 1995.

9. M. L. McConnell and J. M. Ryan, "Status and prospects for polarimetry in high energy astrophysics," New Astronomy Reviews 48, pp. 215-219, 2004.

10. P. F. Bloser, et al., "Development of silicon strip detectors for a medium energy gamma-ray telescope," Nuclear Instruments and Methods in Physics Research A 512, p. 220, 2003.

11. F. Schopper, et al., "CsI calorimeter with 3-D position resolution," Nuclear Instruments and Methods in Physics Research A 442, p. 394, 2000.

12. V. N. Litvinenko and J. M. Madey, "High-power inverse Compton $\gamma$-ray source at the Duke storage ring," in Time-Resolved Electron and X-ray Diffraction, P. M. Rentzepis, ed., Proc. SPIE 2521, pp. 55-77, 1995.

13. R. Andritschke, et al., "The calibration setup of the MEGA prototype at the high intensity $\gamma$-ray source," New Astronomy Reviews 48, pp. 281-285, 2004.

14. A. Zoglauer, R. Andritschke, and G. Kanbach, "Data analysis for the MEGA prototype," New Astronomy Reviews 48, pp. 231-235, 2004.

15. A. Zoglauer, et al., "Imaging properties of the MEGA prototype," in Portland 2003 Nucl. Sci. Symp. Conf. Rec., Proc. IEEE, 2004.

16. S. J. Wilderman, et al., "Fast algorithm for list mode back-projection of Compton scatter cameras," IEEE Transactions on Nuclear Science 45, p. 957, 1998.

17. A. Zoglauer, Methoden der Bildrekonstruktion für das Comptontelekop MEGA, Diploma Thesis (in German), Technical University Munich, 2000.

18. A. Zoglauer, R. Andritschke, and G. Kanbach, "Image reconstruction for the MEGA telescope," in Proceedings of the Fifth INTEGRAL Science Workshop, V. Schönfelder, G. Lichti, and C. Winkler, eds., ESA Special Publication SP-552, p. 917, 2005.

19. M. Marisaldi, et al., "Calorimeter prototype based on silicon drift detectors coupled to scintillators for Compton telescopes application," New Astronomy Reviews 48, p. 305, 2004.

20. J. R. Macri, et al., "Single-sided CZT strip detectors," IEEE Transactions on Nuclear Science 51, p. 2453, 2004.

21. K. S. Shah, et al., "LaBr 3 :Ce scintillators for gamma ray spectroscopy," IEEE Transactions on Nuclear Science 50, p. 2410, 2003. 